\title{
Presuppositions are fast, whether hard or soft - Evidence from the visual world*
}

\author{
Florian Schwarz \\ University of Pennsylvania
}

\begin{abstract}
One focus of work on the processing of linguistic meaning has been the relative processing speed of different aspects of meaning. While much early work has focused on implicatures in comparison to literal asserted content (e.g., Bott \& Noveck 2004; Huang \& Snedeker 2009, among many others), the present paper extends recent efforts to experimentally investigate another aspect of meaning, namely presuppositions. It investigates the triggers again and stop using the visual world eye tracking paradigm, and provides evidence for rapid processing of presupposed content. Our study finds no difference in timing for the two triggers, which is of theoretical relevance given proposals for distinguishing classes of triggers, such as hard vs. soft (Abusch 2010). Whatever differences between these there may be are apparently not affecting the online processing time-course. As a further comparison, again was also compared to twice, which expresses essentially the same meaning without a presupposition. Shifts in eye movements for these two cases also appear to be entirely on par, further supporting the notion that presupposed and asserted content are available in parallel early on in online processing.
\end{abstract}

Keywords: presuppositions, presupposition triggers, presupposition projection, negation, experimental pragmatics, visual world paradigm

\section{Introduction}

When comprehending a sentence uttered in context, hearers quickly arrive at their best guess at what we might call the 'overall conveyed meaning', seemingly without any obvious effort. However, many decades of research on meaning in philosophy of language and linguistics have taught us that this overall meaning is not monolithic, but rather a composite of various different types of ingredients. Since the distinctions between these aspects of meaning are by no means obvious to the naive speaker,

* Thanks to Robert Wilder, Jamie Fisher, Elizabeth Shay and Amy Goodwin-Davies for their assistance with experiment implementation and data collection, and to Dorothy Ahn for providing the visual stimuli. I'd also like to acknowledge my collaborators on various related projects, including Sonja Tiemann, Jacopo Romoli, and Cory Bill. I thank both them and audiences at SALT 24 and CUNY 27 for highly useful discussions of issues related to the present paper. 
it is the job of the theoretician to tease them apart. Key theoretical questions in this regard include a) just what aspects of meaning there are, b) what properties the various classes of meaning have, and c) how they come about. The traditional picture emerging from the extensive theoretical literature, largely building on the seminal work by Paul Grice (Grice 1975), includes four core aspects of meaning, which can be illustrated with the example in (1):

(1) Some of the students have failed the damn exam again.

a. Literal asserted content: a subset of students failed (literal some)

b. Conversational Implicature (scalar): not all of the students failed (some)

c. Presuppositions: this happened before

(again)

d. Conventional Implicature / Expressive:

speaker feels negatively about the exam

(damn)

While the general need for distinctions between classes and even the broad outlines for specific categories of meaning are quite generally established, lively debates continue in the current literature with regards to the exact properties of each class and the details of where the lines between classes are to be drawn. In recent years, the value of experimental approaches to investigating issues related to this has become increasingly evident, as they can help to test and refine theoretically motivated distinctions based on a systematic empirical evaluation of specific expressions. Eventually, this will help to assess the potential need for redrawing some of the traditional boundaries as well as for making more fine-grained distinctions between sub-types of meaning.

On a more general level, experimental approaches also open up novel perspectives by combining insights from formal semantics and pragmatics with perspectives from psychology and cognitive science on the actual mental processes involved in language comprehension. One question of particular interest in this regard concerns the time-course of access to different aspects of meaning, specifically in relation to one another. Uncovering the relative order in which different ingredients of meaning arise in actual processing has the potential to inform the question of how they come about and in what ways they might depend on one another. Ultimately, a comprehensive perspective on how each component of meaning enters the picture and how they all are combined into the overall conveyed meaning will significantly enrich both our theoretical understanding as well as theories of language processing. The perhaps most well-studied point in case, scalar implicatures, can serve as an illustration. The some-but-not-all implicature of some (already illustrated in (1)) is generally assumed to be a pragmatic enrichment of literal content. This could be introduced as a type of default, due to its common presence with some, or, alternatively, may involve an effortful computation on each occurrence. Following Bott \& Noveck 
Presuppositions are fast, whether hard or soft

(2004), there has been a sizable body of evidence in favor of the latter view from various methodologies (including the visual world paradigm, e.g., Huang \& Snedeker 2009). However, more recent results, primarily based on the visual world paradigm (Grodner, Klein, Carbary \& Tanenhaus 2010; Breheny, Ferguson \& Katsos 2013, among others), suggest that implicatures can be rapidly available, at least in some contexts. While the jury is still out on resolving this seemingly conflicting data, the debates over recent years in this area have shown that both theoretical and processing perspectives are greatly enriched by incorporating experimental methodologies in studying these types of phenomena.

The present paper presents experimental investigations of another aspect of meaning, presuppositions, using the visual world paradigm. I begin with a brief review of the theoretical landscape and previous experimental work on presuppositions. Next I present two experiments, which look at the presupposition triggers again and stop respectively. Finally, I discuss possible interpretations of the results in a broader context and provide an outlook on further directions of research, some of which are already well under way.

\section{Background}

\subsection{Theoretical perspectives on presuppositions}

Following the tradition of Stalnaker $(1973,1974,1978)$, presuppositions are commonly taken to have two core properties. First, they generally are already taken for granted by the discourse participants, i.e., they (typically) do not convey new information. Secondly, they display special behavior in various embedding environments, where - unlike asserted content - they are not affected by the embedding operator, but rather 'project' to contribute their content at the global level of the utterance (Karttunen 1973, 1974). Stalnaker's account of presuppositional phenomena is couched in a more general framework that models linguistic exchanges in terms of information updates. The common ground represents information that is mutually accepted by the discourse participants (for the purposes of the conversation), and can be modeled as a set of possible worlds consistent with the propositions established in the discourse. On the simplest view, anything that follows from the common ground counts as presupposed. While Stalnaker's view amounts to an essentially pragmatic perspective, it is clear that there is a range of linguistic expressions that are associated with a presupposition, i.e., in one way or another, they introduce a pre-condition of sorts that the common ground must be of a certain type.

Developments in linguistic semantics in the 1980s (Heim 1982, 1983; Kamp 1981) adapted Stalnaker's perspective by incorporating the notion of context and the potential of expressions to change the context into the semantic system directly. 
Heim's context change semantics reconstrued sentence meanings precisely in terms of context change potentials. Presuppositions then can be seen as definedness conditions on updating a given context with a sentence. Such an approach can also account for projection phenomena, based on suitable assumptions for the update procedures associated with the relevant embedding operators (Heim 1983). Within Discourse Representation Theory (DRT; Kamp 1981), a similarly dynamic approach is taken, but with an additional representational layer to keep track of the cumulative information in a discourse explicitly. Accounts of presuppositions in this framework generally see them as a form of anaphora, which require resolution to some previously introduced piece of information in the discourse representation structure (van der Sandt \& Geurts 1991; van der Sandt 1992; Geurts 1999).

While dynamic approaches represent a more semantic view on presuppositions, pragmatic accounts in a Stalnakerian spirit have recently seen a revival, with various proposals for assimilating the analysis of at least certain types of presupposition triggers to that of scalar implicatures (Abusch 2002, 2010; Romoli 2011). Schlenker (2009) lays out a slightly different pragmatic approach, which essentially attempts to recast the insights from Heim's theory in non-dynamic terms, based on the (technical) notion of a 'Local Context'. This (and related proposals) is of particular interest from a processing perspective, as it aims to capture presupposition projection in terms of more general processing factors related to incremental interpretation.

In addition to these theoretical developments at a general level, various authors have argued for the need to differentiate between different types of triggers, because of differences in their behavior, e.g., with respect to their relation to the context, their projection behavior, and their availability for accommodation. An early proposal along these lines was made by Zeevat (1992), who distinguishes resolution triggers (e.g., again) from lexical triggers (e.g., stop), based on the extent to which the triggers rely directly on preceding expressions, and to what extent their presupposition is tied up with the asserted meaning of the trigger. More recently, Abusch (2002, 2010) has distinguished 'soft' and 'hard' triggers (for other proposals for distinctions between triggers, see Jayez 2013; Tiemann 2014). In the following, I will use Abusch's distinction, though the two expressions to be investigated will largely fall on opposing sides on most classification schemes (including Zeevat's), so the choice is primarily a terminological one for the ease of presentation. To illustrate the contrast briefly, one of the key differences between hard and soft triggers, according to Abusch, concerns the extent to which their global impact can be suspended.

(2) I don't know whether John ever played golf...

a. \# But if he played golf again, ...

b. $\checkmark$ But if he stopped playing golf, ...

The context sentence here is set up so as to be inconsistent with the following 
Presuppositions are fast, whether hard or soft

presupposition triggers. The hard trigger again in (2a) seems to lead to infelicity in this context, while the presupposition of the soft trigger stop in (2b) can be suspended (and perhaps instead be interpreted inside of the antecedent of the conditional). ${ }^{1}$ Abusch $(2002,2010)$ proposes an account of this difference by deriving soft triggers based on reasoning about alternative expressions (see also Romoli 2014, who treats soft triggers as implicatures).

\subsection{Experimental results on presuppositions}

Turning to experimental work on presuppositions, some of the crucial questions are very much on par with those investigated in the experimental literature on implicatures. In particular, in both cases, there are ongoing theoretical debates about the status of the relevant inferences, which can be seen as either semantic (i.e., encoded as part of the lexical content of certain expressions or structures) or pragmatic (e.g., based on general reasoning). This question can be related to the issue of the processing time-course of the relevant inferences: if the availability of an inference is delayed relative to asserted content, that is commonly seen as evidence in favor of pragmatic accounts. We will utilize parallel reasoning in our experimental comparison between presupposition triggers. Beyond this issue of processing time-course, presuppositions give rise to a host of additional questions that are equally intriguing. For example, there is the question of how people reject sentences with unsatisfied presuppositions (Syrett, Koev, Angelides \& Kramer 2014; Schwarz 2014b). Furthermore, the interaction of presuppositions with both the intraand extra-linguistic context, e.g., in presupposition projection, has rich potential for experimental investigations (Chemla \& Schlenker 2012; Schwarz \& Tiemann 2012, 2013a). And last but not least, a detailed assessment of differences between presupposition triggers has much to gain from experimental methodologies. In the following, I provide a very brief review of some of the existing results directly relevant to the present study. For a more comprehensive state-of-the-art report on the current literature, see Schwarz 2014c (as well as the contributions to Schwarz 2014a).

Among the earliest attempts at assessing the time-course of presupposition processing in comprehension is a set of reading studies based on manipulating the felicity of a sentence containing a trigger in the presented context. For example, Schwarz (2007) used contextual manipulations such as the following (slashes indicate region boundaries in moving-window self-paced reading):

a. The congressman / who wrote to John / ...

1 See Jayez, Mongelli, Reboul \& van der Henst 2014 for data that call into question the notion that the contrast between hard and soft triggers is indeed as solid as Abusch presents it. 
b. The congressman / who John wrote to / ...

$\ldots$ had also written to the mayor / ...

The presupposition of also (here that the congressman had written to someone other than the mayor as well, assuming default stress) is satisfied in the first variant but not the second. Tiemann, Schmid, Bade, Rolke, Hertrich, Ackermann, Knapp \& Beck (2011) extend this approach to other triggers using word-by-word self-paced reading (see also Tiemann, Kirsten, Beck, Hertrich \& Rolke 2014; Tiemann 2014), and Schwarz \& Tiemann (2012) use eye tracking during reading on similar stimuli. The general finding is that when the context is inconsistent with the presupposition introduced by a trigger, this leads to (more or less) immediate slow-downs in reading. This is attributed to the detection of the inconsistency, which in turn is indicative of the availability of the presupposition.

More recently, various authors have begun to systematically compare presupposition triggers to one another using behavioral measures. For example, Cummins, Amaral \& Katsos 2013 and Amaral \& Cummins 2014 find differences in acceptability of responses to sentences containing triggers that challenge the presupposition and begin with yes, but... or no, because..., and argue that Zeevat's lexical triggers generally are more compatible with the latter than resolution triggers. With respect to the soft vs. hard distinction introduced by Abusch, Jayez et al. (2014) argue that even a hard trigger such as too can be accommodated in the antecedent of conditionals. But other authors report further data in support of differences between triggers (e.g., Smith \& Hall 2011; Velleman, Beaver, Bumford, Destruel \& Onea 2011; Destruel, Onea, Velleman, Bumford \& Beaver 2014). While these behavioral data have an important role to play in better understanding individual triggers and the relationship between them, it is also desirable to have more direct online measures on the various triggers in order to compare their online processing properties. The studies below present a first effort in this direction.

\section{3 again and stop in the visual world paradigm}

\subsection{Aims \& general design}

The experiments below were designed to provide insights into the online processing time-course of the triggers again and stop, both in relationship to one another and, in case of the former, relative to asserted content. As these two triggers are commonly classified as belonging to different groups of triggers such as hard and soft, respectively, this will also inform the more general question of whether there are corresponding differences in online processing. The paradigm employed is the visual world eye tracking paradigm (Tanenhaus, Spivey-Knowlton, Eberhard \& Sedivy 1995), which makes it possible to observe unfolding interpretations in close 
Presuppositions are fast, whether hard or soft

to real time. Unlike the reading time studies mentioned above, it does not require presentation of presuppositions in infelicitous contexts, as it can provide evidence for the availability of a given interpretation while listening to felicitous sentences and relating them to a visual context. The experimental task and stimuli are kept as parallel as possible for both experiments to maximize comparability of the results for the two triggers.

As is standard in visual world studies, we measured the timing of fixations on different parts of a display relative to the unfolding auditory stimulus. The task was to identify the individual that the sentence was about. During a certain time window, only the presupposed inference made it possible to home in on one individual. Shifts in fixations during that time window are indicative of the availability of the inference. In addition to allowing us to track the shifting focus of attention throughout hearing the linguistic stimuli, the visual world paradigm has the advantage of not requiring any conscious decisions on the part of the subject. Eye movements are largely beyond our conscious control, and the crucial role of the presupposed information lasted only very briefly, as additional information introduced later on in the sentence provided independent disambiguation. This reduced the risk of participants noticing the nature of the experimental manipulation and developing explicit strategies that could introduce effects that are artifacts of the task, rather than features of the regular comprehension process.

\subsection{Experiment 1: again vs. twice}

Design The first experiment looked at the trigger again, which roughly presupposes that an event of the sort described in the clause that it adjoins to has previously taken place and is sufficiently salient in the discourse. In order to gain a comparison to the time-course of processing asserted content, we compared again to twice, which also conveys that some type of event has occurred more than once, but without any presuppositionality involved. Since they also can occur in the same position in the sentence, this affords us a rather minimal comparison between presupposed and asserted content. In terms of methodology, we adapted the visual world paradigm from studies of similar phenomena such as implicatures (e.g., Sedivy 2003; Huang $\&$ Snedeker 2009), using a $2 \times 2$ interaction design. The first manipulation varied whether one and the same crucial inference was presupposed or asserted. The other manipulation was implemented by varying the pictures in the visual display, which affected whether or not the target could be identified early on in the sentence during an otherwise ambiguous period.

The visual displays included four characters: a target, a competitor, and two opposite gender distractors. The task explained to the subjects in an introductory screen was that they would have to decide which of the characters the sentence was 


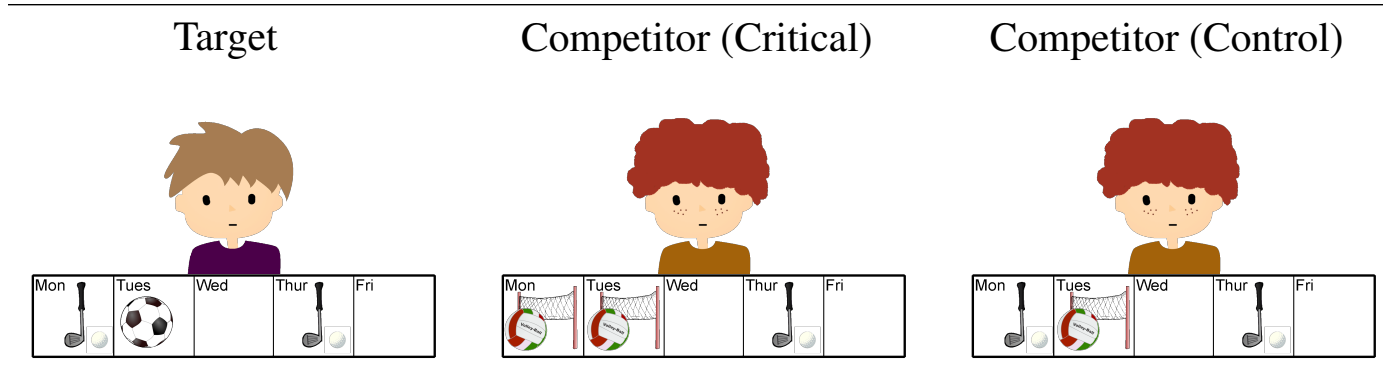

Figure 1 Again/Twice visual stimuli.

about. To differentiate the individuals, we used a calendar strip paradigm, with five boxes representing days of the week underneath each person. Some of the boxes contained iconic representations of activities or food items. An illustration is provided in Figure 1. A context sentence such as the one in (4a) was used to set the scene and to ensure the felicity of the use of again/twice in the target sentence (here by highlighting that there were golf and volleyball events at the beginning of the week). The target sentences made it possible to identify which one of the depicted figures the sentence is about (e.g., which one is John).

a. Context: Some of these children went to play golf on Monday, and some to play volleyball.

b. Target: John went to play golf... i. again later on ...

ii. twice this week ...

$\ldots$ and also played soccer on Tuesday.

Crucially, based on the choice of competitor picture, the point at which the target could be identified varied. In the control condition, this was not possible until the word soccer occurred near the end of the sentence, since only the target picture involved a soccer event. In the critical condition, on the other hand, the target could already be identified while hearing the underlined part of the sentence, starting with again or twice, but only if the crucial inference that the relevant individual had played golf at an earlier point in the week is available right away, as this is precisely what distinguishes the critical competitor from the target. Thus, any shift in fixations towards the target during the time window corresponding to that part of the sentence indicates that subjects are able to access this inference. Note, however, that the critical inference was not necessary for selecting the target at a global level, as the later phrase involving soccer provided independent disambiguation at the end of the 
Presuppositions are fast, whether hard or soft

sentence. This was intended to prevent subjects from becoming aware of the critical manipulation and the impact of the expressions of interest.

Participants, materials \& procedure 27 students from the University of Pennsylvania participated in the experiment. 24 items parallel to those illustrated above were constructed, with versions in each of the four conditions. Items were counterbalanced across participants, who each saw 6 items per condition. In addition to the experimental items, there were 24 other items from an independent experiment that served as fillers. For each item, recordings of the relevant sentences were created. Identical recordings of the again/twice-versions of the target sentence were used for the critical and control conditions, and the same recording of the context sentence was used for all four conditions. The visual stimuli were set up so that both the target and the competitor always involved repetition of one of the types of events at the beginning of the week in order to avoid the possibility of identifying targets based on features of the visual stimuli alone. For similar reasons, the activity mentioned in the target sentence corresponded to the first mentioned activity in the context sentence in half of the items, and to the second mentioned one in the other half. At the beginning of the experiment, participants were seated in front of a computer and an EyeLink 1000 eye tracker, which was calibrated to track the participant's right eye. They then saw an introductory screen with basic instructions and an illustration of the types of pictures they would see, and subsequently performed a practice trial. Each trial began with a fixation cross displayed in the middle of the screen. Next, the visual display was shown for 3000ms before playback of the auditory recording began, so that participants could familiarize themselves with the pictures shown. The trials ended when the participant clicked on the picture they thought the sentence was about, and were followed by a break of $1000 \mathrm{~ms}$ prior to moving on to the next trial.

Data \& Analysis The main dependent measure consisted of the proportion of looks to the target picture during the period of interest, which began 200ms after the onset of again/twice and ended 200ms after the onset of the independently disambiguating expression (e.g., soccer) (the 200ms shift is due to the time it takes to program and execute a saccade). Time-locking eye-movement data to the onset of the critical word allows us to attribute the unfolding shift in fixations to the impact of the inference of interest. Rather than analyzing plain proportions of fixations to the target, we computed Target Advantage scores by subtracting looks to the competitor from looks to the target. This relative measure provides a direct perspective on any potential preference for the target, reflected in positive measures ( 0 being the baseline reflecting equal amounts of looks to target and competitor). The graph in Figure 2 illustrates target advantage scores for all four conditions. 


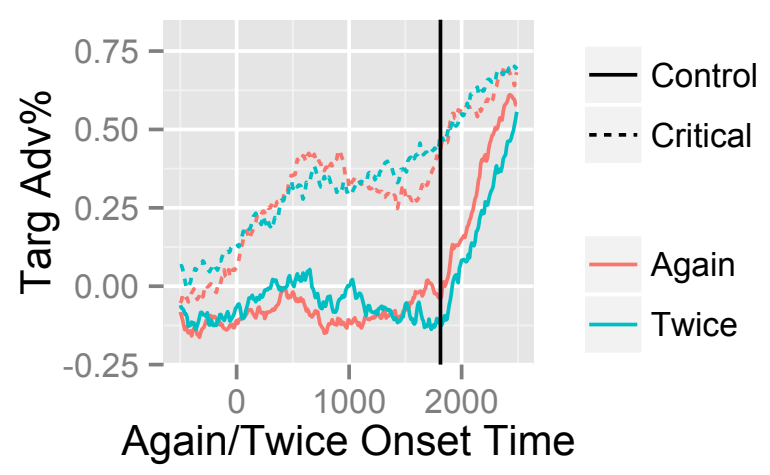

Figure 2 Target Advantage Scores across conditions. 0 marks the onset of again / twice. Vertical line indicates mean onset of independent disambiguation.

Data from the control conditions (solid lines) hovers at zero, reflecting equal likelihood of looking at target and competitor prior to the independent disambiguation (indicated by the vertical line). As can be seen from the sharp rise right around the onset of again/twice in the critical conditions (dashed lines), fixations are shifting towards the target quickly and remain biased towards the target throughout the otherwise ambiguous period. There is no apparent difference between the again and twice conditions. To assess the effects statistically, we computed overall Target Advantage scores for the ambiguous time window and transformed them into empirical logits (Barr 2008). Analyses used mixed-effect models with subjects and items as random effects, using the lmer function of the lme 4 package in $R$ (Bates 2005). Following Barr, Levy, Scheepers \& Tily (2013), the maximal random effect structure that would converge was used in each case, with a random intercept as well as random slopes for both main effects and the interaction. Full random effect structures converged and were used unless otherwise noted. To assess whether inclusion of a given factor significantly improved the fit of the overall model, likelihood-ratio tests were performed that compared two minimally different models, one with the fixed effects factor in question and one without, while keeping the random effects structure identical (Barr et al. 2013). We report estimates, standard errors, and $t$-values for all models, as well as the $\chi^{2}$ and $p$-value from the likelihood-ratio test for individual factors.

A $2 \times 2$ interaction model of the data revealed a significant main effect of the critical vs. control manipulation $\left(\beta=4.78, S E=0.62, t=7.64 ; \chi^{2}=33.28, p<\right.$ $.001)$, but no main effect for again vs. twice and no interaction $\left(p^{\prime} s>.8\right)$. To get a more detailed perspective on the time-course of the shift in fixations, we conducted the same analysis for data from the first $200 \mathrm{~ms}$ of the ambiguous time window 
Presuppositions are fast, whether hard or soft

(i.e., 200-400ms after the onset of twicelagain). This yielded the same result, with a significant main effect of critical vs. control $(\beta=3.28, S E=0.79, t=4.15$; $\left.\chi^{2}=12.89, p<.001\right)$ and no other significant effects. ${ }^{2}$ Planned comparisons for both the full ambiguous time window and its first 200ms further confirmed that the effect of critical vs. control was significant for both the again- and twice-subsets of the data. To address potential concerns that participants' behavior adapts to the nature of the stimuli over the course of the experiment, which could lead to overestimating the speed with which the relevant inferences are available, additional analyses including trial order within each session were conducted. The trial order coefficients were not significantly different from zero and inclusion of this factor did not significantly increase the models (for either the full time window or the first $200 \mathrm{~ms}$ ). Thus, there is no evidence of practice effects leading to adaptation and increased predictability throughout the course of the experiment.

Discussion The rapid increase in fixations on the target following the onset of both again and twice indicates that the key inference these expressions contribute (e.g., that John had played golf before in the week) is immediately available in online processing. Furthermore, given that one of the expressions, namely again, introduces this information as part of the presupposed content, this provides evidence for the immediate availability of presupposed content in online processing. The comparison with twice, which introduces the same inference but as part of the asserted content, provides the most direct comparison of these two aspects of meaning to date with regards to online processing. Both types of content seem to be available in parallel, in contrast with (some of the) findings on scalar implicatures.

\subsection{Experiment 2: stop}

Design The second experiment extended the paradigm described above to investigate presupposed inferences associated with the verb stop, namely that the activity described by the complement verb phrase used to take place (whereas it asserts that it is not taking place now). As noted in the introduction, stop has been characterized as a soft trigger, and its presupposition analyzed in terms of reasoning about alternatives (Abusch 2010) or as a type of implicature (Romoli 2014). Given previous findings in the literature that implicated content is delayed relative to literal, asserted content (e.g., Huang \& Snedeker 2009), such an analysis gives rise to the question of how quickly the presupposed inference introduced by stop is available in online processing.

2 As the model with the maximal random effects structure did not converge, the interaction term was removed from the random effects structure for items. 


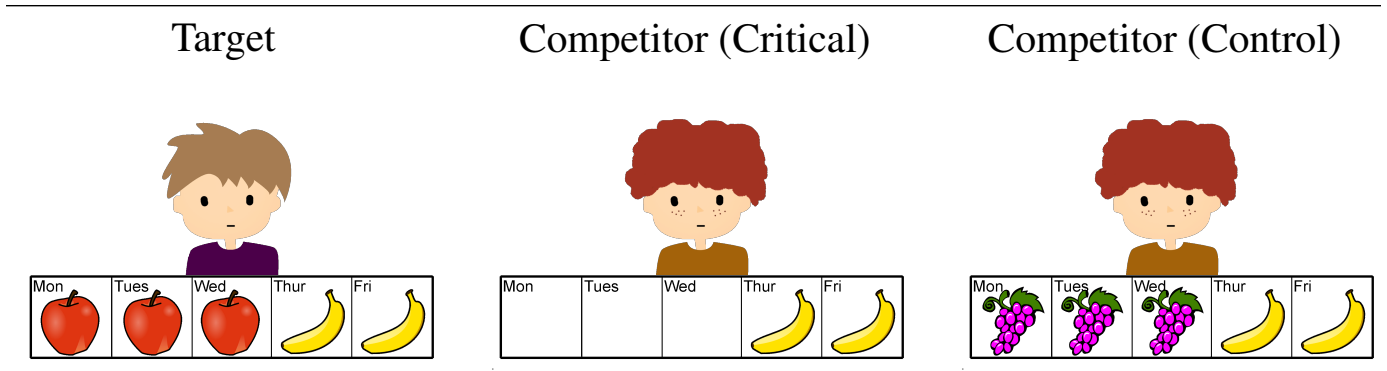

Figure 3 Visual stimuli for experiment on stop.

The calendar-strip paradigm was adapted for investigating this issue, by creating sequences of like activities that could be described as stopping at some point. For example, the target and competitor pictures in Figure 3 were paired with the context and target sentences in (5):

a. Context: These children got nice treats for their snacks this week.

b. Target: John stopped eating the delicious apples on Thursday.

The use of stop in the target sentence introduces the presupposition that John had been eating apples at an earlier point of the week, which is only true of the target character. However, prior to the realization of the word apples, the sentence is equally compatible with the control competitor, who had been eating grapes earlier in the week. Thus, apples is the point of disambiguation when comparing these two pictures. In the critical condition, on the other hand, the competitor had no activities at the beginning of the week, and thus cannot be described as stopping any activity later on. In this case, it is precisely the presupposition of stop that makes it possible to identify the target right upon hearing the trigger, prior to encountering apples (i.e., during the time window corresponding to the underlined portion of the sentence). As before, independent disambiguation is provided towards the end of the sentence (here at the point of apples, just as in the control condition).

Participants, materials and procedure 27 students from the University of Pennsylvania participated in the experiment. Critical items consisted of 24 pairs of recordings of sentences and pictures along the lines illustrated above. Each item came in four versions, based on the competitor picture manipulation and an additional manipulation of whether or not negation was included in the sentence. For the moment, we will focus on the affirmative items only. (Results for negated items will be discussed below.) Each participant saw 6 items per condition, with counterbalancing across participant groups. In addition to the critical items, there were 24 


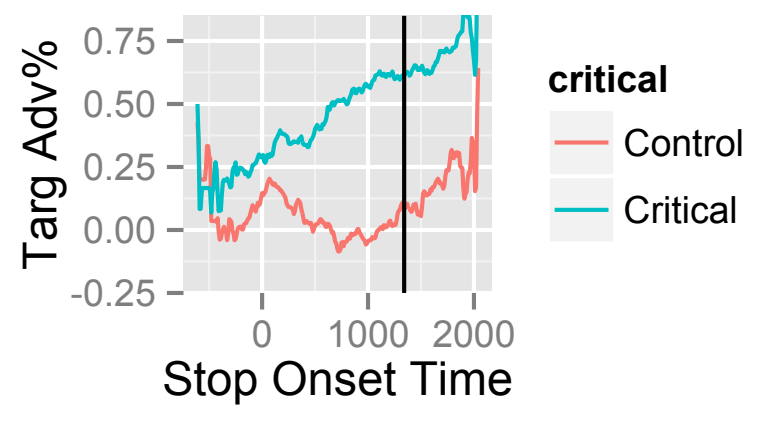

Figure 4 Target Advantage Scores by conditions. 0 marks the onset of stop. Vertical line indicates mean onset of independent disambiguation.

items from another sub-experiment that served as fillers, as well as 24 genuine filler items that addressed various potential counter-balancing concerns. Unlike in the previous experiment, the display included only the target and competitor, i.e., there were no additional gender distractors. The general procedure and instructions were parallel to the first experiment. Due to the more limited number of figures within the display, preview time before playback of the auditory recording was set to $2000 \mathrm{~ms}$.

Data \& Analysis Data analysis proceeded as in the first experiment, with a focus on target advantage scores for the time window corresponding to the underlined part of the target sentence, which was ambiguous in the control condition and potentially disambiguated by the presupposed inference introduced by stop. The graph in Figure 4 illustrates target advantage scores by condition.

Around the onset of stop, there is a shift in fixations to the target in the critical condition, whereas no consistent bias is present in the control condition. As in the first experiment, we assessed this difference statistically using mixed-effect models run on elogits computed from the target advantage scores. Looking at the entire time window of interest (from 200ms after the onset of stop until 200ms after the onset of the disambiguating word, such as apples), there was a significant difference between conditions, with greater target advantage scores in the critical condition ( $\left.\beta=5.27, S E=0.75, t=6.99 ; \chi^{2}=10.1, p<.01\right)$. Looking at just the first $200 \mathrm{~ms}$ of this time window revealed that the effect was already present early on after the onset of stop $\left(\beta=3.23, S E=1.07, t=3.02 ; \chi^{2}=8.98, p<.01\right)$. As in the first experiment, we also constructed corresponding models with trial order as a factor, but this did not significantly improve the model, providing evidence against any significant practice effects that are relevant for the results of interest. 
Discussion The immediate shift in fixations towards the target after encounter of the verb stop in the critical condition indicates that the presupposition of stop (e.g., that John had been eating apples earlier in the week) is immediately available in online processing, as it provides the only possible piece of information that can help to identify the target picture. As such, the results found here are entirely on par with those for again in the first experiment. Assuming that an interpretation along these lines can be maintained, this suggests that the soft and hard triggers looked at here do not differ in their online processing time-course. However, there are some further considerations we need to address, which we turn to in the next section.

\section{General discussion \& further issues}

\subsection{Entailed presuppositions?}

Throughout the discussion of the two experiments above, we have assumed that the inferences of again and stop in question are unequivocally presuppositional. However, there is a common view in the literature that at least in the case of certain presupposition triggers, the relevant inferences have a dual status of sorts, and are entailed as well as presupposed. From such a perspective, evidence for the availability of the relevant content is not necessarily indicative of the time-course of presupposed content, as it might simply be a reflex of its status as an entailment. Not all triggers are equally suitable for such a line of argument. Recent discussion by Sudo (2012), for example, suggests that only the presuppositions of some triggers are also entailed. While a more detailed assessment of his criteria for determining this with respect to the triggers under investigation here is ultimately required, again seems likely to pattern with those triggers that do not entail their presupposition, in contrast with stop. This, of course, would have a direct impact on the interpretation of our results, as it leaves open the possibility that the presupposition of the former is indeed available immediately, whereas in the latter case, the immediate reflex of the relevant inference is merely due to the fact that it is also entailed. This would be a step towards making the results more consistent with analyses of soft triggers in terms of alternatives or implicatures.

However, there is at least some initial evidence that speaks against attributing the rapid availability of the presupposition of stop to its putative additional status as an entailment. First, the entailment point becomes moot in the scope of negation, where only the presupposition survives at the global level. Nonetheless, recent evidence suggests that stop and again display parallel processing properties under negation as well (see Schwarz \& Tiemann 2013b, and data presented below). To the extent that this holds, that speaks against analyzing the effects found here in different terms for the two triggers we looked at. 
Presuppositions are fast, whether hard or soft

Assertion: False

Presupposition: True

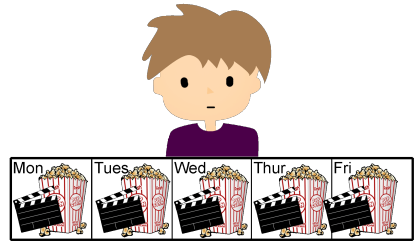

Assertion: ??

Presupposition: False

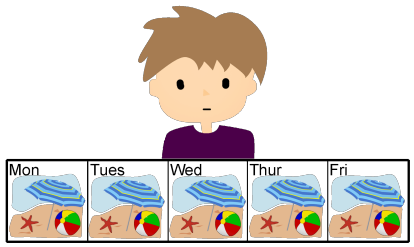

Figure 5 Overt picture choices for Covered Box study on stop.

Even on a unified analysis of the data reported here, one could still maintain, of course, that both are instances of triggers whose presupposition is also entailed. However, recent findings on response times related to presupposed content from our lab provide tentative evidence to the contrary. In particular, we have found that rejections of sentences based on presupposed content are slower than rejections based on asserted content. This has been reported for definite descriptions (Schwarz 2014b), but also seems to hold for the verb stop. In a study carried out with Cory Bill and Jacopo Romoli, we used a covered box picture selection task to assess the availability of various interpretations of stop in different contexts. Participants had to choose whether a given sentence matched an overtly shown one or one that was hidden from view instead, under the assumption that only one of the two would match the sentence. One of the contrasts we included varied whether the overtly shown picture violated the presupposed or the asserted content of stop. For example, the sentence in (6) would be paired with either one of the pictures in Figure 5, along with an alternative covered box choice.

(6) John stopped going to the movies on Wednesday.

The picture on the left is one that should be rejected based on asserted content alone, since the presupposition (that John had been going to the movies earlier) clearly is met. For the picture on the right, our perspective on the basis for rejection depends on whether we assume the presupposition to double as entailed content. If it does, then the rejection here could be based on either type of content (or possibly both). Interestingly, while rejection rates (in the form of covered box choices) are at ceiling for both conditions, response times for rejections based on asserted content alone are significantly faster than rejections of the picture on the right (2692ms vs. $3562 \mathrm{~ms}$ ). This is more compatible with the view that the presupposition does not feature in what is entailed as well, in particular if we assume that any evidence for rejection is acted on as soon as it is encountered (for evidence that can be argued to support this view, see Schwarz 2014b). For the picture on the 


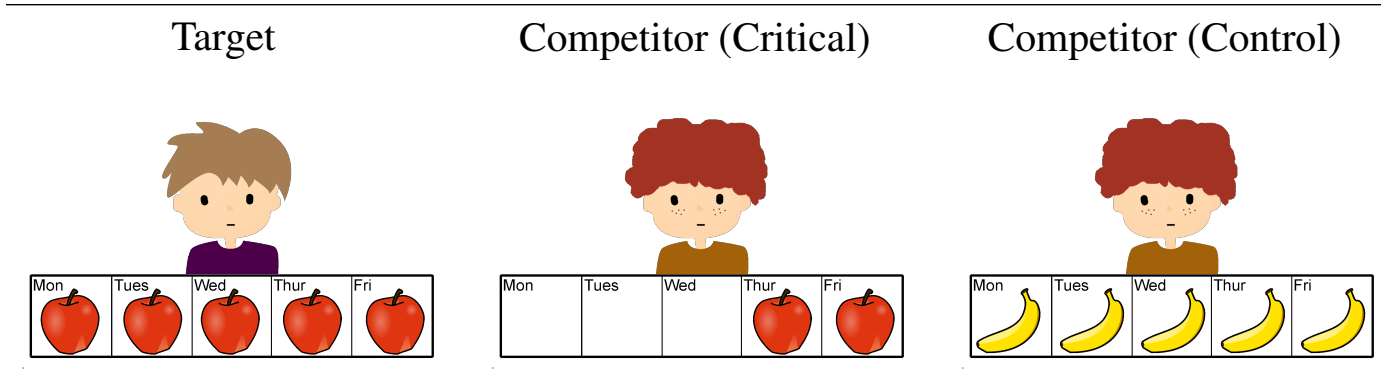

Figure 6 Visual stimuli for negated conditions in the experiment on stop.

right could also be rejected based on asserted content alone if the presupposition indeed features as an entailment of the sentence. So why should it matter that it is additionally presupposed, if a decision can be made based on what is asserted alone? Interestingly, the same pattern can be found in negated cases as well, whereas mentioned above - the issue of entailment becomes moot. This again would seem to speak against seeing affirmative and negated cases as involving different circumstances with respect to the status of the presupposed content. While it may be possible to understand this data from a perspective that sees the presupposition of stop as part of what is entailed as well, these results lend at least tentative support to the view that they are not, since this accounts for the difference in response times straightforwardly.

\subsection{Presupposition projection: don't stop}

As already mentioned, there is further evidence for stop and again to behave in parallel ways under negation, which bears on the issue of whether their presuppositions might be entailed in affirmative cases, and furthermore whether they might differ from one another in that regard. Schwarz \& Tiemann (2012, 2013b) provide evidence from reading time studies on German wieder ('again') for a processing cost of presupposition projection under negation and in conditionals. In brief, detecting inconsistencies of the presupposition with the discourse context seems to take longer when the presupposition trigger is introduced in an embedded environment. As part of the visual world study on stop reported above, we also included conditions with stop appearing in the scope of negation. Using the same logic as laid out above, we paired pictures like those in Figure 6 with recordings of the sentences in (7).

(7) a. Context: These children got nice treats for their snacks this week.

b. Target: Henry didn't stop eating the delicious apples all week. 
Presuppositions are fast, whether hard or soft

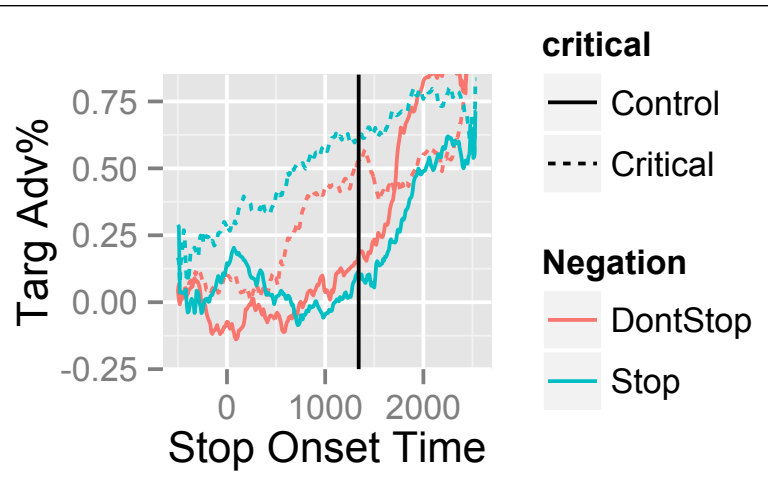

Figure 7 Target Advantage Scores by condition, including negated stop items. 0 marks the onset of stop. Vertical line indicates mean onset of independent disambiguation.

Just as in the affirmative version, the presupposition of stop makes it possible to identify the target in the critical condition upon the encounter of stop, whereas independent disambiguation is necessary in the control condition.

Target advantage scores for both affirmative and negated conditions are plotted in Figure 7. While the negated condition does show an increase in fixations to the target prior to the independent disambiguation, this does not seem to happen as quickly as in the affirmative condition. While space constraints prevent me from presenting a detailed statistical analysis, the most crucial finding is that there is a significant interaction in the ambiguous time-window $\left(\beta=2.28, S E=1.26, t=1.82 ; \chi^{2}=\right.$ $5.67, p<.05)$, which seems to be due to the temporary difference in relation between control and critical conditions for affirmative and negated versions respectively. This preliminary finding then suggests that projection of the presupposition of stop is associated with a delay comparable to that reported by Schwarz \& Tiemann (2012, 2013 b) for again. This, in turn, further supports the view that the equivalent finding of rapid availability of presupposed content of again and stop (in affirmative contexts) in the present studies does not come about in different ways, but rather does constitute evidence for the immediate presence of presupposed content (whether introduced by a hard or a soft trigger) once a trigger is encountered.

\subsection{Conclusion and outlook}

We have considered evidence from two visual world experiments on the presupposition triggers again and stop, which are commonly argued to belong to different sub-classes of triggers, with potentially different mechanisms giving rise to the 
relevant inferences. Our data suggests that in both cases, the presupposed content is available rapidly. In the case of again, we furthermore provided a direct comparison with essentially the same content when asserted, in the form of twice, and found no difference in the time-course of the inference becoming available. These findings are in line with previous visual world findings for also (Romoli, Khan, Snedeker \& Sudo 2014; Schwarz 2014d). They enhance our understanding of the time-course of presupposition processing beyond what was known from previous reading time evidence, in that they provide a more detailed level of temporal resolution while allowing us to observe the natural unfolding of the interpretation process in fully felicitous contexts.

The present results suggest that there are no differences between the soft and hard triggers we looked at when it comes to processing time-course, but of course there may be other grounds for differentiating classes of triggers that are independent of that. In line with previous findings, the immediate availability of presupposed content speaks against views that would assume presuppositions to arise through pragmatic inferencing that is costly, and therefore delayed, in terms of online processing. They are fully compatible with semantic views of presuppositions that take them to be conventionally encoded as part of the lexical entries of the relevant expressions. But they are also compatible with pragmatic proposals if we assume that the relevant type of pragmatic content is rapidly available, as has been argued for other cases in various other studies within the visual world paradigm.

On a more general level, the present studies provide a proof of concept of sorts, in that they show that we can use the powerful psycholinguistic tools employed here to study detailed aspects of presupposition interpretation and related theoretical questions. Much of the work in this area still lies ahead. For one, it will be crucial to gain further evidence on whether presuppositions should also be seen as entailed, at least for certain triggers, since this plays a crucial role for the interpretation of the present data, as reviewed above. Further issues, several of which we have already begun to pursue in our lab (largely in collaboration with Cory Bill and Jacopo Romoli), include a more direct comparison of presuppositions and implicatures, in particular with respect to embedding under negation; a closer look at presuppositionrelated behavior in different populations, such as young children and populations with language-related disorders; and investigation of other embedding environments, e.g., embedding under attitude verbs. With the tools at hand, the prospect for theoretical insights from empirical investigations along these lines seems promising.

\section{References}

Abusch, Dorit. 2002. Lexical alternatives as a source of pragmatic presuppositions. In Brendan Jackson (ed.), Semantics and Linguistic Theory (SALT) 12, 1-19. Ithaca, 
Presuppositions are fast, whether hard or soft

NY: Cornell University. http://elanguage.net/journals/salt/article/view/12.1.

Abusch, Dorit. 2010. Presupposition triggering from alternatives. Journal of Semantics 27(1). 37 -80. doi:10.1093/jos/ffp009.

Amaral, Patricia \& Chris Cummins. 2014. A cross-linguistic study on information backgrounding and presupposition projection. In Florian Schwarz (ed.), Experimental Perspectives on Presuppositions, 157-172. Berlin: Springer.

Barr, Dale J. 2008. Analyzing 'visual world' eyetracking data using multilevel logistic regression. Journal of Memory and Language 59(4). 457-474. doi:10.1016/j.jml.2007.09.002.

Barr, Dale J., Roger Levy, Christoph Scheepers \& Harry J. Tily. 2013. Random effects structure for confirmatory hypothesis testing: Keep it maximal. Journal of Memory and Language 68(3). 255-278. doi:10.1016/j.jml.2012.11.001.

Bates, Douglas M. 2005. Fitting linear mixed models in R. R News 5. 27-30.

Bott, Lewis \& Ira A. Noveck. 2004. Some utterances are underinformative: The onset and time course of scalar inferences. Journal of memory and language 51(3). 437-457. doi:10.1016/j.jml.2004.05.006.

Breheny, Richard, Heather J. Ferguson \& Napoleon Katsos. 2013. Investigating the timecourse of accessing conversational implicatures during incremental sentence interpretation. Language and Cognitive Processes 28(4). 443-467. doi:10.1080/01690965.2011.649040.

Chemla, Emmanuel \& Philippe Schlenker. 2012. Incremental vs. symmetric accounts of presupposition projection: an experimental approach. Natural Language Semantics 20(2). 177-226. doi:10.1007/s11050-012-9080-7.

Cummins, Chris, Patricia Amaral \& Napoleon Katsos. 2013. Backgrounding and accommodation of presupposition: an experimental approach. In Emanuel Chemla, Vincent Homer \& Gregoire Winterstein (eds.), Sinn und Bedeutung (SuB) 17, 201-218. http://semanticsarchive.net/sub2012/: Semanticsarchive.

Destruel, Emilie, Edgar Onea, Dan Velleman, Dylan Bumford \& David Beaver. 2014. A cross-linguistic study of the non-at-issueness of exhaustive inferences. In Florian Schwarz (ed.), Experimental Perspectives on Presuppositions, 135-156. Berlin: Springer.

Geurts, Bart. 1999. Presuppositions and Pronouns. Amsterdam; New York: Elsevier. Grice, H.P. 1975. Logic and conversation. In Peter Cole \& Jerry L. Morgan (eds.), Syntax and Semantics 3, 41-58. New York: Academic Press.

Grodner, Daniel J., Natalie M. Klein, Kathleen M. Carbary \& Michael K. Tanenhaus. 2010. "Some," and possibly all, scalar inferences are not delayed: Evidence for immediate pragmatic enrichment. Cognition 116(1). 42-55. doi:10.1016/j.cognition.2010.03.014.

Heim, Irene. 1982. The semantics of definite and indefinite noun phrases: University of Massachusetts Amherst $\mathrm{PhD}$ thesis. 
Heim, Irene. 1983. On the projection problem for presuppositions. In M. Barlow, D. Flickinger \& N. Wiegand (eds.), West Coast Conference on Formal Linguistics (WCCFL) 2, 114-125. Stanford University.

Huang, Yi Ting \& Jesse Snedeker. 2009. Online interpretation of scalar quantifiers: Insight into the semantics-pragmatics interface. Cognitive psychology 58(3). 376-415. doi:10.1016/j.cogpsych.2008.09.001.

Jayez, Jacques. 2013. Presupposition triggers and orthogonality. Ms. Lyon.

Jayez, Jacques, Valeria Mongelli, Anne Reboul \& Jean-Baptiste van der Henst. 2014. Weak and strong triggers. In Florian Schwarz (ed.), Experimental Perspectives on Presuppositions, 173-194. Berlin: Springer.

Kamp, Hans. 1981. A theory of truth and semantic representation. In Jeroen Groenendijk, Theo M.V. Janssen \& Martin Stokhof (eds.), Amsterdam Colloquium (AC) 2, 227-321. Amsterdam: Mathematical Center.

Karttunen, Lauri. 1973. Presuppositions of compound sentences. Linguistic Inquiry 4(2). 169-193.

Karttunen, Lauri. 1974. Presupposition and linguistic context. Theoretical Linguistics 1. 181-194.

Romoli, Jacopo. 2011. The presuppositions of soft triggers aren't presuppositions. In Neil Ashton, Anca Chereches \& David Lutz (eds.), Semantics and Linguistic Theory (SALT) 21, 236-256. Ithaca, NY: CLC Publications. http://elanguage. net/journals/salt/article/view/21.236.

Romoli, Jacopo. 2014. The presuppositions of soft triggers are obligatory scalar implicatures. Journal of Semantics (Advance Access). doi:10.1093/jos/fft017.

Romoli, Jacopo, Manizeh Khan, Jesse Snedeker \& Yasutada Sudo. 2014. Resolving temporary referential ambiguity using presupposed content. In Florian Schwarz (ed.), Experimental Perspectives on Presuppositions, 67-88. Berlin: Springer.

van der Sandt, Rob. 1992. Presupposition projection as anaphora resolution. Journal of Semantics 9. 333-377. doi:10.1093/jos/9.4.333.

van der Sandt, Rob \& Bart Geurts. 1991. Presupposition, anaphora, and lexical content. In O. Herzog \& C.-R. Rollinger (eds.), Text Understanding in LILOG, 259-296. Berlin: Springer.

Schlenker, Philippe. 2009. Local contexts. Semantics and Pragmatics 2. doi:10.3765/sp.2.3.

Schwarz, Florian. 2007. Processing presupposed content. Journal of Semantics 24(4). 373-416. doi:10.1093/jos/ffm011.

Schwarz, Florian (ed.). 2014a. Experimental Perspectives on Presuppositions. Berlin: Springer.

Schwarz, Florian. 2014b. False but slow: Evaluating statements with non-referring definites. Ms., UPenn.

Schwarz, Florian. 2014c. Introduction: Presuppositions in context - theoretical 
Presuppositions are fast, whether hard or soft

issues and experimental perspectives. In Florian Schwarz (ed.), Experimental Perspectives on Presuppositions, 1-38. Berlin: Springer.

Schwarz, Florian. 2014d. Presuppositions vs. asserted content in online processing. In Florian Schwarz (ed.), Experimental Perspectives on Presuppositions, 89-108. Berlin: Springer.

Schwarz, Florian \& Sonja Tiemann. 2012. Presupposition processing - the case of German wieder. In Maria Aloni, Vadim Kimmelmann, Floris Roelofsen, Galit W. Sassoon, Katrin Schulz \& Matthijs Westera (eds.), Amsterdam Colloquium (AC) 18, 200-209. Berlin: Springer.

Schwarz, Florian \& Sonja Tiemann. 2013a. The path of presupposition projection in processing - the case of conditionals. In Emanuel Chemla, Vincent Homer \& Gregoire Winterstein (eds.), Sinn und Bedeutung (SuB) 17, 509-526. Semantics Archive. http://semanticsarchive.net/sub2012/.

Schwarz, Florian. \& Sonja. Tiemann. 2013b. Presupposition projection in online processing. (Ms., submitted).

Sedivy, Julie. C. 2003. Pragmatic versus form-based accounts of referential contrast: Evidence for effects of informativity expectations. Journal of Psycholinguistic Research 32(1). 3-23. doi:10.1023/a:1021928914454.

Smith, E. Allyn \& Kathleen C. Hall. 2011. Projection diversity: Experimental evidence. Workshop on Projective Meaning at ESLLI 2011.

Stalnaker, Robert. 1973. Presuppositions. Journal of Philosophical Logic 2(4). 447-457. doi:10.1007/bf00262951.

Stalnaker, Robert. 1974. Pragmatic presuppositions. In Milton K. Munitz \& Peter K. Unger (eds.), Semantics and Philosophy, 197-213. New York: New York University Press.

Stalnaker, Robert. 1978. Assertion. In Peter Cole (ed.), Syntax and Semantics 9, 315-322. New York: Academic Press.

Sudo, Yasutada. 2012. On the semantics of phi features on pronouns. Cambridge, MA: Massachusetts Institute of Technology PhD thesis.

Syrett, Kristen, Todor Koev, Nicholas Angelides \& Maxwell Kramer. 2014. Experimental evidence for the truth conditional contribution and shifting information status of appositives. Journal of Semantics (Online first). doi:10.1093/jos/ffu007.

Tanenhaus, Michael K., Michael J. Spivey-Knowlton, Kathleen M. Eberhard \& Julie C. Sedivy. 1995. Integration of visual and linguistic information in spoken language comprehension. Science 268(5217). 1632-1634. doi:10.1126/science. 7777863 .

Tiemann, Sonja. 2014. The processing of wieder ('again') and other presupposition triggers. Tübingen: Eberhard Karls Universität Tübingen $\mathrm{PhD}$ thesis.

Tiemann, Sonja, Mareike Kirsten, Sigrid Beck, Ingro Hertrich \& Bettina Rolke. 2014. Presupposition processing and accommodation: An experiment on wieder 
('again') and consequences for other triggers. In Florian Schwarz (ed.), Experimental Perspectives on Presuppositions, 39-66. Berlin: Springer.

Tiemann, Sonja, Mareike Schmid, Nadine Bade, Bettina Rolke, Ingo Hertrich, Hermann Ackermann, Julia Knapp \& Sigrid Beck. 2011. Psycholinguistic evidence for presuppositions: On-line and off-line data. In Ingo Reich, Eva Horch \& Dennis Pauly (eds.), Sinn und Bedeutung (SuB) 15, 581-597. Münster: Monsenstein.

Velleman, Dan, David Beaver, Dylan Bumford, Emilie Destruel \& Edgar Onea. 2011. "yes, but..." - exhaustivity and at-issueness across languages. Poster presented at PEPA 2011.

Zeevat, Henk. 1992. Presupposition and accommodation in update semantics. Journal of Semantics 9(4). 379-412. doi:10.1093/jos/9.4.379.

Florian Schwarz

619 Williams Hall

University of Pennsylvania

255 S. 36th Street

Philadelphia, PA 19104-6305

florians@ling.upenn.edu 